\title{
CONSISTENCY THEOREMS FOR ALMOST CONVERGENCE
}

\author{
BY \\ G. BENNETT( ${ }^{1}$ ) AND N. J. KALTON
}

\begin{abstract}
The concept of almost convergence of a sequence of real or complex numbers was introduced by Lorentz, who developed a very elegant theory. The purpose of the present paper is to continue Lorentz's investigations and obtain consistency theorems for almost convergence; this is achieved by studying certain locally convex topological vector spaces.
\end{abstract}

1. Introduction The concept of almost convergence of a sequence of real or complex numbers was introduced, after an idea of Banach, by Lorentz [13] who developed a very elegant theory. Further studies of almost convergence and its relationship with general summability methods have since been carried out in [12], [17] and [19]. The purpose of the present paper is to obtain consistency theorems for almost convergence by studying certain locally convex topological vector spaces.

We adopt the following notation:

$\omega$ denotes the space of all scalar (real or complex) sequences;

$e, e^{(k)} \in \omega$ are given by

$$
\begin{aligned}
e & =(1,1, \ldots), \\
e^{(k)} & =(0, \ldots, 0,1,0, \ldots) \quad \text { with the one in the } k \text { th position; }
\end{aligned}
$$

$\varphi$ is the linear span of $\left\{e^{(k)}: k=1,2, \ldots\right\}$;

$m=\left\{x \in \omega:\|x\|_{\infty}=\sup _{j}\left|x_{j}\right|<\infty\right\}$;

$c=\left\{x \in \omega: \lim x=\lim _{j \rightarrow \infty} x_{j}\right.$ exists $\}$

$c_{0}=\{x \in \omega: \lim x=0\}$

$l=\left\{x \in \omega:\|x\|_{\mathrm{h}}=\sum_{j=1}^{\infty}\left|x_{j}\right|<\infty\right\}$;

$b v=\left\{x \in \omega:\|x\|_{b v}=\sum_{j=1}^{\infty}\left|x_{j}-x_{j+1}\right|+\lim _{j \rightarrow \infty}\left|x_{j}\right|<\infty\right\}$;

$b v_{0}=b v \cap c_{0}$;

$b s=\left\{x \in \omega:\|x\|_{b s}=\sup _{n}\left|\sum_{j=1}^{n} x_{j}\right|<\infty\right\}$.

A vector subspace of $\omega$ is called a sequence space. If $E$ is a sequence space with a locally convex topology $\tau$ then $(E, \tau)$ is a $K$-space provided that the linear functionals

Received by the editors December 14, 1972.

AMS (MOS) subject classifications (1970). Primary 46A45, 40H05; Secondary 46A05, 40C05, 40D20.

Key words and phrases. Banach limit, almost convergence, topological sequence space, FK-space, matrix transformation, consistency theorems, superconvergence in topological vector spaces.

(1) During the preparation of this manuscript the first named author was supported in part by NSF grant GP 33694. 


$$
x \rightarrow x_{j} \quad(j=1,2, \ldots)
$$

are continuous on $E$. If, in addition, $(E, \tau)$ is complete and metrizable (respectively normable) then $(E, \tau)$ is called an $F K$-space (respectively $B K$-space). For $x \in \omega$ we write

$$
P_{n} x=\left(x_{1}, x_{2}, \ldots, x_{n}, 0, \ldots\right) .
$$

$(E, \tau)$ is an $A K$-space if $P_{n} x$ converges to $x$ for every $x \in E$.

If $E$ and $F$ are sequence spaces containing $\varphi$ such that the bilinear form $\langle x, y\rangle=\sum_{j=1}^{\infty} x_{j} y_{j}$ converges whenever $x \in E$ and $y \in F$, then topologies of the dual pairing $\langle E, F\rangle$ provide examples of $K$-space topologies. In particular, we shall be interested in the weak topology $\sigma(E, F)$, the Mackey topology $\tau(E, F)$ and the strong topology $\beta(E, F)$ (following the notation of Schaefer [18]).

We shall also consider matrix maps and matrix methods of limitation. Let $A=\left(a_{i j}\right)_{i, j=1}^{\infty}$ be an infinite matrix with scalar entries; we denote by $\omega_{A}$ the set of $x \in \omega$ such that $\sum_{j=1}^{\infty} a_{i j} x_{j}$ converges for each $i$. For $x \in \omega_{A}$ we write

$$
(A x)_{i}=\sum_{j=1}^{\infty} a_{i j} x_{j}
$$

so that $A: \omega_{A} \rightarrow \omega$ is a linear map. If $E$ is a sequence space,

$$
E_{A}=\left\{x \in \omega_{A}: A x \in E\right\} .
$$

If $E$ is an $F K$-space then Zeller [24, Theorem 4.10(a)] has shown that $E_{A}$ is also an $F K$-space when topologized by means of the seminorms:

$$
\begin{array}{ll}
x \rightarrow x_{j} & (j=1,2, \ldots), \\
x \rightarrow \sup _{n}\left|\sum_{j=1}^{n} a_{i j} x_{j}\right| & (i=1,2, \ldots),
\end{array}
$$

and

$$
x \rightarrow q(A x),
$$

where $q$ runs through the continuous seminorms on $E$. A matrix $A$ defines a method of limitation, viz: if $x \in c_{A}$, we write $\lim _{A} x=\lim (A x) . A$ is called conservative if $c \subseteq c_{A}$ or, equivalently (see [26]),

$$
\begin{aligned}
\sup _{i} \sum_{j=1}^{\infty}\left|a_{i j}\right| & <\infty, \\
\lim _{i \rightarrow \infty} a_{i j} & =a_{j} \text { exists } \quad(j=1,2, \ldots),
\end{aligned}
$$

and

$$
\lim _{i \rightarrow \infty} \sum_{j=1}^{\infty} a_{i j} \text { exists. }
$$


We then write

$$
\chi(A)=\lim _{i \rightarrow \infty} \sum_{j=1}^{\infty} a_{i j}-\sum_{j=1}^{\infty} a_{j},
$$

and say that $A$ is conull when $\chi(A)=0 . A$ is called regular if $\lim _{A} x=\lim x$ whenever $x \in c$; for regularity it is necessary and sufficient (see [26]) to have (1), (2) and (3) with $a_{j}=0(j=1,2, \ldots)$ and $\chi(A)=1$.

2. Properties of almost convergence. In this section we develop the theory of almost convergence, deriving the original characterization of almost convergent sequences given by Lorentz [13], as well as several other useful properties of the space $a c_{0}$ (to be defined below). Since our approach is from the viewpoint of functional analysis, and therefore differs slightly from Lorentz's, we shall give a complete development of the subject.

The linear functional lim on $c$ has norm one, i.e.

$$
|\lim x| \leq\|x\|_{\infty} \quad(x \in c)
$$

and so by the Hahn-Banach theorem possesses extensions $L$, of norm one, defined on all of $m$. We call such a functional $L$ an extended limit. If $x \in \omega$, we write

$$
T x=\left\{x_{n+1}\right\}_{n=1}^{\infty}
$$

and say that an extended limit $L$ is a Banach limit if

$$
L(T x)=L(x) \quad(x \in m) .
$$

(Some authors insist that a Banach limit should also satisfy $L(x) \geq 0$ whenever $x_{n} \geq 0$ for all $n$, or even $\lim _{n \rightarrow \infty} \sup x_{n} \geq L(x) \geq \lim _{n \rightarrow \infty}$ inf $x_{n}$. It is clear, however, that any extended limit has these properties.)

The existence of Banach limits was proved by Banach [2]; another proof can be found in Theorem 1 below. If $x \in m$ is such that for every Banach limit $L$, $L(x)$ assumes a common value, then we write $\operatorname{Lim} x$ for this value, and say that $x$ is almost convergent to $\operatorname{Lim} x$. The set of almost convergent sequences is denoted by $a c$, and the subset $\{x \in a c: \lim x=0\}$ is denoted by $a c_{0} . a c_{0}$ is a hyperplane in $a c$ and $a c=a c_{0}+\{e\}$; it is also easy to show that $a c$ and $a c_{0}$ are closed supspaces of $m$. Our first result (Theorem 1 ) characterizes these spaces.

Lemma 1. If $L$ is a continuous linear functional on $\boldsymbol{m}$ with

(i) $\|L\|=1$,

(ii) $L(e)=1$, and

(iii) $L(b s)=0$,

then $L$ is a Banach limit.

Proof. Since $\varphi \subseteq b s$, it follows from (iii) that $L(\varphi)=0$, and by continuity that 
$L\left(c_{0}\right)=0$; therefore $L$ is an extended limit. Moreover, for $x \in m, x-T x \in b s$ and so $L(x)=L(T x)$.

Lemma 2. If $x \in m \mid c_{0}$, then there exists an extended limit $L$ with $L(x) \neq 0$.

Proof. Since $x \in m \mid c_{0}$, we may choose an increasing sequence $\left\{n_{k}\right\}_{k=1}^{\infty}$ of positive integers such that

$$
\lim _{k \rightarrow \infty} x_{n_{k}}=\alpha \neq 0
$$

Define $L$ by

$$
L y=\lim _{k \rightarrow \infty} y_{n_{k}}
$$

where this limit exists, and extend $L$ to $m$ by the Hahn-Banach theorem.

Theorem 1 (Lorentz) [13] $x \in \omega$ is almost convergent (to $\alpha)$ if and only if

$$
\lim _{p \rightarrow \infty} \frac{1}{p}\left(x_{n}+\cdots+x_{n+p-1}\right)=\alpha
$$

uniformly in $n$.

Proof. Without loss of generality we may assume that $\alpha=0$. Let $\left\{n_{p}\right\}_{p=1}^{\infty}$ be any increasing sequence of positive integers, and define the matrix map $A: m$ $\rightarrow m$ by

$$
(A x)_{p}=\frac{1}{p}\left(x_{n_{p}}+\cdots+x_{n_{p}+p-1}\right) \quad(x \in m) .
$$

Then we have $A e=e, A(b s) \subset c_{0},\|A\|_{\infty}=1$.

If $L$ is an extended limit, then, by Lemma 1 ,

$$
L A \text { is a Banach limit }
$$

and so, for $x \in a c_{0}$, we have

$$
L(A x)=0 .
$$

By Lemma 2 we have $A x \in c_{0}$ so that

$$
\lim _{p \rightarrow \infty} \frac{1}{p}\left(x_{n_{p}}+\cdots+x_{n_{p}+p-1}\right)=0 .
$$

Since this is true for any sequence $\left\{n_{p}\right\}_{p=1}^{\infty}$, we conclude that

$$
\lim _{p \rightarrow \infty} \sup _{n}\left|\frac{1}{p}\left(x_{n}+\cdots+x_{n+p-1}\right)\right|=0,
$$

which is (4).

Conversely, (4) implies that 


$$
\lim _{p \rightarrow \infty}\left\|\frac{1}{p}\left(T x+\cdots+T^{p} x\right)\right\|_{\infty}=0 .
$$

Thus, for any Banach limit $L$, we have $L(x)=0$, so that $x \in a c_{0}$.

We remark that (5) gives what is perhaps the easiest proof of the existence of Banach limits. Banach's original proof [2] also uses the Hahn-Banach theorem, but involves a rather sophisticated sublinear functional; Day's elegant proof $[9$, p.83], using fixed point theory, requires considerably more machinery.

Our next result, which follows at once from Theorem 1 , shows that $a c_{0}$ and $a c$ are "large" subspaces of $m$.

Corollary. $\left(a c_{0},\|\cdot\|_{\infty}\right)$ is a nonseparable $B K$-space.

We now come to a series of results which relate various properties of $a c_{0}$ to those of more familiar sequence spaces.

Theorem 2. If $\left\{x^{(n)}\right\}_{n=1}^{\infty}$ is a sequence of points in l, and $x \in I$, then the following conditions are equivalent:

(i) $\left\{x^{(n)}\right\}_{n=1}^{\infty}$ is $\sigma\left(l, a c_{0}\right)$-convergent to $x$ :

(ii) $\left\{x^{(n)}\right\}_{n=1}^{\infty}$ is $\sigma\left(l, b s+c_{0}\right)$-convergent to $x$;

(iii) $\sup _{n}\left\|x^{(n)}\right\|_{h}<\infty$ and $\lim _{n \rightarrow \infty}\left\|x^{(n)}-x\right\|_{b v}=0$.

Proof. Without loss of generality we may assume that $x=0$.

(i) $\Rightarrow$ (ii) follows since $b s+c_{0} \subset a c_{0}$.

(ii) $\Rightarrow$ (iii). If $x^{(n)} \rightarrow 0 \sigma\left(l, b s+c_{0}\right)$, then $x^{(n)} \rightarrow 0 \sigma\left(l, c_{0}\right)$ so that

$$
\sup _{n}\left\|x^{(n)}\right\|_{h}<\infty \text {. }
$$

Also, $x^{(n)} \rightarrow 0 \sigma(l, b s)$ so that $x^{(n)} \rightarrow 0 \sigma\left(b v_{0}, b s\right)$; this is the weak topology on $b v_{0}$, and, since $b v_{0}$ is isomorphic to $l$, we may use Schur's theorem [2, p. 137] to deduce that

$$
\lim _{n \rightarrow \infty}\left\|x^{(n)}\right\|_{b v}=0 .
$$

(iii) $\Rightarrow$ (i). Let $f \in a c_{0}$ and $\varepsilon>0$ be fixed. By Theorem 1 we may choose a positive integer $p$ so that

$$
\left\|\frac{1}{p}\left(T f+\cdots+T^{p} f\right)\right\|_{\infty}<\varepsilon / 2\left(1+\sup _{n}\left\|x^{(n)}\right\|_{1}\right) .
$$

We then have, for every $n$,

$$
\begin{aligned}
& \left|\sum_{k=1}^{\infty} \frac{1}{p}\left(f_{k+1}+\cdots+f_{k+p}\right) x_{k}^{(n)}\right| \\
& \quad \leq\left\|x^{(n)}\right\|_{1}\left\|\frac{1}{p}\left(T f+\cdots+T^{p} f\right)\right\|_{\infty}<\frac{\varepsilon}{2} .
\end{aligned}
$$

Furthermore, fixing $p$,we may choose a positive integer $N$ so that 


$$
\left\|x^{(n)}\right\|_{b v}<\frac{\varepsilon}{2(p+1)\left(1+\|f\|_{\infty}\right)}
$$

whenever $n \geq N$.

Now

$$
\begin{aligned}
\left|\sum_{k=1}^{\infty}\left(f_{k+s}-f_{k}\right) x_{k}^{(n)}\right| & \left.=\left|\sum_{k=1}^{\infty} f_{k}\left(x_{k-s}^{(n)}-x_{k}^{(n)}\right)\right| \quad \text { (putting } x_{m}^{(n)}=0 \text { if } m \leq 0\right) \\
& \leq \sum_{k=1}^{\infty}\left|f_{k}\right| \sum_{r=1}^{s}\left|x_{k-r+1}^{(n)}-x_{k-r}^{(n)}\right| \\
& \leq s\|f\|_{\infty}\left(\left\|x^{(n)}\right\|_{b v}+\left|x_{1}^{(n)}\right|\right) \\
& \leq 2 s\|f\|_{\infty}\left\|x^{(n)}\right\|_{b v} .
\end{aligned}
$$

Therefore

$$
\begin{aligned}
\left|\sum_{k=1}^{\infty} \frac{1}{p}\left(f_{k+1}+\cdots+f_{k+p}\right) x_{k}^{(n)}-\sum_{k=1}^{\infty} f_{k} x_{k}^{(n)}\right| & \leq \frac{1}{p} \frac{p(p+1)}{2} 2\|f\|_{\infty}\left\|x^{(n)}\right\|_{b v} \\
& <\frac{\varepsilon}{2} \text { whenever } n \geq N
\end{aligned}
$$

Thus, for $n \geq N$, we have

$$
\left|\sum_{k=1}^{\infty} f_{k} x_{k}^{(n)}\right|<\varepsilon
$$

i.e., $x^{(n)} \rightarrow 0 \sigma\left(l, a c_{0}\right)$.

We remark that condition (iii) of Theorem 2 identifies sequential convergence in $\sigma\left(l, a c_{0}\right)$ with a two-norm topology. For details concerning this type of topology we refer the reader to [1], [6], [22] and [23].

Corollary $1 . l$ is sequentially complete under both the topologies $\sigma\left(l, a c_{0}\right)$ and $\sigma\left(l, b s+c_{0}\right)$.

Proof. If $\left\{x^{(n)}\right\}_{n=1}^{\infty}$ is a $\sigma\left(l, b s+c_{0}\right)$-Cauchy sequence, the proof of Theorem 2 shows that $\left\{x^{(n)}\right\}_{n=1}^{\infty}$ is a Cauchy sequence in $b v_{0}$ and bounded in $l$. Since $b v_{0}$ is complete, there exists $x \in b v_{0}$ such that

$$
\lim _{n \rightarrow \infty}\left\|x^{(n)}-x\right\|_{b v}=0
$$

But this implies that

$$
x_{k}=\lim _{n \rightarrow \infty} x_{k}^{(n)} \quad(k=1,2, \ldots)
$$

so that

$$
\sum_{k=1}^{\infty}\left|x_{k}\right| \leq \sup _{n} \sum_{k=1}^{\infty}\left|x_{k}^{(n)}\right|<\infty
$$


and $x \in l$. It then follows from Theorem 2, (iii) $\Rightarrow(\mathrm{i})$, that $x^{(n)} \rightarrow 0 \sigma\left(l, a c_{0}\right)$, giving the desired result.

Corollary 2. For a subset $C$ of $l$, the following conditions are equivalent:

(i) $C$ is $\sigma\left(l, a c_{0}\right)$-relatively compact;

(ii) $C$ is $\sigma\left(l, b s+c_{0}\right)$-relatively compact;

(iii) $C$ is $\|\cdot\|_{1}$-bounded and $\lim _{n \rightarrow \infty} \sup _{x \in C}\left\|x-P_{n} x\right\|_{b v}=0$.

Proof. A subset of a $K$-space is relatively compact if and only if it is relatively sequentially compact (see [10]) and hence Theorem 2 shows that (i) and (ii) are equivalent. Using the sequential completeness of $l$ in the two-norm convergence defined in (iii) of Theorem 2, it is clear that (i) and (ii) are equivalent to " $C$ is $\|\cdot\|_{1}$-bounded and $\|\cdot\|_{b_{0}}$-relatively compact." However, by a general theorem on bases (see [16]) this is equivalent to (iii).

We note from Corollary 2 that the closed convex hull of a $\sigma\left(l, a c_{0}\right)$-compact set is also $\sigma\left(l, a c_{0}\right)$-compact (using (iii)); hence the Mackey topology, $\tau\left(a c_{0}, l\right)$, is the topology of uniform convergence on $\sigma\left(l, a c_{0}\right)$-compact sets.

We now turn to the relationship between $a c_{0}$ and $b s$.

Theorem 3. (i) $a c_{0}=\overline{b s}$, the closure of bs in $m$.

(ii) If $x \in b s+c_{0}$, then $\sup _{p} \lim \sup _{n \rightarrow \infty}\left|x_{n+1}+\cdots+x_{n+p}\right|<\infty$.

(iii) $a c_{0} \neq b s+c_{0}$.

Proof. (i) Clearly $\overline{b s} \subseteq a c_{0}$. Conversely, if $x \in a c_{0}$ and $\varepsilon>0$ are given, we may choose a positive integer $p$ so that

$$
\left|x_{n+1}+\cdots+x_{n+p}\right|<p \varepsilon \quad(n=1,2, \ldots) .
$$

In particular,

$$
x_{m p+1}+\cdots+x_{(m+1) p}=p \delta_{m} \quad(m=0,1,2, \ldots)
$$

where $\left|\delta_{m}\right| \leq \varepsilon$. Letting $y$ be defined by

$$
y_{m p+k}=x_{m p+k}-\delta_{m} \quad(k=1,2, \ldots, p ; m=0,1,2, \ldots),
$$

it is clear that $\|x-y\|_{\infty} \leq \varepsilon$; we complete the proof of (i) by showing that $y \in b s$.

Now

$$
\begin{aligned}
\sum_{i=1}^{m p+k} y_{i} & =\sum_{n=0}^{m-1} \sum_{j=1}^{p}\left(x_{n p+j}-\delta_{n}\right)+\sum_{i=1}^{k} x_{m p+i}-k \delta_{m} \\
& =\sum_{i=1}^{k} x_{m p+i}-k \delta_{m} \quad \text { by }(6) .
\end{aligned}
$$

Consequently 


$$
\left|\sum_{i=1}^{q} y_{i}\right| \leq p\left(\|x\|_{\infty}+\varepsilon\right)
$$

for every $q$, and $y \in b s$.

(ii) If $x \in b s+c_{0}$, then $x=y+z$ for some $y \in b s$ and $z \in c_{0}$. Then

$$
\left|x_{n+1}+\cdots+x_{n+p}\right| \leq\left|y_{n+1}+\cdots+y_{n+p}\right|+\left|z_{n+1}+\cdots+z_{n+p}\right|
$$

so that

$$
\limsup _{n \rightarrow \infty}\left|x_{n+1}+\cdots+x_{n+p}\right|=\limsup _{n \rightarrow \infty}\left|y_{n+1}+\cdots+y_{n+p}\right| \leq 2\|y\|_{b s},
$$

giving the desired result.

(iii) By (ii) we may construct $x \in a c_{0} \backslash\left(b s+c_{0}\right)$ directly; let

$$
\begin{aligned}
x_{k} & =1 & & \text { if } k=2^{n}+2^{m} \text { for } n \geq m \geq 1, \\
& =0 & & \text { otherwise } .
\end{aligned}
$$

Then $x$ does not satisfy (ii), yet it is easy to check that $x \in a c_{0}$.

It is interesting to note that $b s+c_{0}$ is a $B K$-space which is $B$-invariant in the sense of Garling [10], and $c_{0} \subset b s+c_{0} \subset m$, yet $b s+c_{0}$ is not closed in $m$.

Theorem 4. (i) $\left(a c_{0}, \tau\left(a c_{0}, l\right)\right)$ is a complete $A K$-space.

(ii) $\tau\left(b s+c_{0}, l\right)$ is the restriction of $\tau\left(a c_{0}, l\right)$ to bs $+c_{0}\left[s o\right.$ that $\left(a c_{0}, \tau\left(a c_{0}, l\right)\right)$ is the completion of $\left.\left(b s+c_{0}, \tau\left(b s+c_{0}, l\right)\right)\right]$.

Proof. (i) If $C$ is $\sigma\left(l, a c_{0}\right)$-relatively compact, then by (iii) of Corollary 2 to Theorem 2, the set $P(C)=\left\{P_{n} f: f \in C\right\}$ is $\sigma\left(l, a c_{0}\right)$-relatively compact. It follows that the operators $\left\{P_{n}: n=1,2, \ldots\right\}$ are $\tau\left(a c_{0}, l\right) \rightarrow \tau\left(a c_{0}, l\right)$-equicontinuous, so that the set

$$
S=\left\{x \in a c_{0}: P_{n} x \rightarrow x \tau\left(a c_{0}, l\right)\right\}
$$

is $\tau\left(a c_{0}, l\right)$-closed. However, $S \supset \varphi$ and $\varphi$ is $\tau\left(a c_{0}, l\right)$-dense in $a c_{0}$ (since $\varphi$ is $\sigma\left(a c_{0}, l\right)$-dense); hence $S=a c_{0}$, showing that $\left(a c_{0}, \tau\left(a c_{0}, l\right)\right)$ is an $A K$-space.

To show that $\left(a c_{0}, \tau\left(a c_{0}, l\right)\right)$ is complete we use Grothendieck's criterion [6, Proposition 1]. Let $\theta$ be a linear functional on $l$ which is $\sigma\left(l, a c_{0}\right)$-continuous on each $\sigma\left(l, a c_{0}\right)$-compact set. Then $\theta\left(x^{(n)}\right) \rightarrow 0$ whenever $x^{(n)} \rightarrow 0 \sigma\left(l, a c_{0}\right)$. Consequently, from Theorem $2, \theta$ is continuous in the two-norm topology. Using the standard characterization of the dual of a two-norm space [1, Theorem 4.2], it follows that $\theta$ lies in the closure of $b s$ (the dual of $\left(l,\|\cdot\|_{b v}\right)$ ) in $m$ (the dual of $\left.\left(l,\|\cdot\|_{\infty}\right)\right)$. Hence, by Theorem 3(i), $\theta$ takes the form

$$
\theta(x)=\sum_{k=1}^{\infty} f_{k} x_{k},
$$

where $f$ is a fixed element from $a c_{0}$. It follows from Grothendieck's criterion that $\left(a c_{0}, \tau\left(a c_{0}, l\right)\right)$ is complete. 
(ii) This follows from Corollary 2 to Theorem 2.

Theorem 5. Let $E$ be a separable FK-space containing $c_{0}$ and $b s$. Then

(i) $E$ contains $a c_{0}$;

(ii) $x \in a c_{0}$ implies that $P_{n} x \rightarrow x$ in $E$;

(iii) $e^{(n)} \rightarrow 0$ in $E$.

Proof. (i) and (ii). The space ( $\left.b s+c_{0}, \tau\left(b s+c_{0}, l\right)\right)$ is a Mackey space whose dual, $l$, is $\sigma\left(l, b s+c_{0}\right)$-sequentially complete by Corollary 1 to Theorem 2 . Thus, by the main result of [11] (see also [7, Theorem 5]), the natural inclusion mapping: $b s+c_{0} \rightarrow E$, which clearly has closed graph, must be continuous. If $x \in a c_{0}$, then by Theorem 4, $\left\{P_{n} x\right\}_{n=1}^{\infty}$ is Cauchy in $\left(b s+c_{0}, \tau\left(b s+c_{0}, l\right)\right)$ and hence in $E$. Since $E$ is complete, $\left\{P_{n} x\right\}_{n=1}^{\infty}$ converges in $E$, and its limit must be $x$ since $E$ is a $K$-space. This completes the proof of (i) and (ii).

For (iii), we note that if $C$ is a $\sigma\left(l, b s+c_{0}\right)$-compact subset of $l$, then

$$
\sup _{f \in C}\left|f_{n}\right| \leq \sup _{f \in C}\left\|f-P_{n-1} f\right\|_{b v} \rightarrow 0 \text { as } n \rightarrow \infty
$$

by Corollary 2 to Theorem 2 . Consequently $e^{(n)} \rightarrow 0 \tau\left(b s+c_{0}, l\right)$ and hence in E.

We note that (iii) is true if we only assume that $E$ contains $b s$ (see [5, Theorem 5]).

Our next result answers a question left open in [6].

Corollary 1. There exists a BK-space $E$ which is not the intersection of the separable FK-spaces containing it.

Proof. Using Theorems 3 and 5 we may take $E$ to be $b s+c_{0}$.

Corollary 2. If $A$ is a conservative matrix such that bs $\subset c_{A}$, then

(i) $a c \subset c_{A}$;

(ii) $\lim _{n \rightarrow \infty} \sup _{m}\left|a_{m n}\right|=0$;

(iii) $\lim _{m \rightarrow \infty} \sup _{n}\left|a_{m n}\right|=0$;

(iv) for $x \in a c$, we have $\lim _{A} x=\sum_{j=1}^{\infty} a_{j} x_{j}+\chi(A) \operatorname{Lim} x$.

Proof. (i) $c_{A}$ is a separable $F K$-space ([14, 1.4.1]; [4, Corollary 1 to Theorem 4]) and so, by Theorem 5(i), $a c_{0} \subset c_{A}$. Since $e \in c_{A}$, it follows that $a c \subset c_{A}$.

(ii) $e^{(n)} \rightarrow 0$ in $c_{A}$ by Theorem 5(iii), so that $A e^{(n)} \rightarrow 0$ in $c$ by Theorem 4.4(c) of [24]. It follows that

$$
\lim _{n \rightarrow \infty} \sup _{m}\left|a_{m n}\right|=0 .
$$

(iii) follows from (ii) as in the proof of Proposition 8 of [5].

(iv) If $x \in a c$, then $x-(\operatorname{Lim} x) e \in a c_{0}$ and, by Theorem 5(ii),

$$
x-(\operatorname{Lim} x) e=\sum_{k=1}^{\infty}\left(x_{k}-\operatorname{Lim} x\right) e^{(k)} \text { in } c_{A} .
$$

Now $\lim _{A}$ is continuous on $c_{A}$ so that 


$$
\lim _{A} x-(\operatorname{Lim} x) \lim _{A} e=\sum_{k=1}^{\infty}\left(x_{k}-\operatorname{Lim} x\right) a_{k} .
$$

Since $x \in m, \sum_{k=1}^{\infty} a_{k} x_{k}$ converges and so

$$
\begin{aligned}
\lim _{A} x & =\sum_{k=1}^{\infty} a_{k} x_{k}+\operatorname{Lim} x\left(\lim _{A} e-\sum_{k=1}^{\infty} a_{k}\right) \\
& =\sum_{k=1}^{\infty} a_{k} x_{k}+\chi(A) \operatorname{Lim} x .
\end{aligned}
$$

3. Consistency theorems. In [6] we used a technique involving the Orlicz-Pettis theorem on unconditional convergence of series to obtain a new proof of the Mazur-Orlicz-Brudno consistency theorem. In this section we apply the same basic technique to derive similar consistency theorems for almost convergence; the details, however, are much more difficult than those in [6] and we shall need considerable preparation before coming to our main results (Theorems 6 and 8).

We begin by introducing an idea which may be of some interest in a more general setting; we say that a sequence $\left\{x^{(n)}\right\}_{n=1}^{\infty}$ is superconvergent to $x$ (in a locally convex space $E$ ) if $\left\{x^{(n)}\right\}_{n=1}^{\infty}$ converges to $x$ and

$$
\sum_{k=1}^{\infty}\left(x_{n_{k}}-x_{n_{k}-1}\right)
$$

converges in $E$ for every increasing sequence $\left\{n_{k}\right\}_{k=1}^{\infty}$ of positive integers.

Our first result is elementary and its proof is omitted.

Lemma 3. Every subsequence of a superconvergent sequence is superconvergent.

The validity of the next result is one of the main reasons for studying superconvergence.

Lemma 4. Let $E$ be a locally convex space with dual $E^{\prime}$. If a sequence $\left\{x^{(n)}\right\}_{n=1}^{\infty}$ superconverges in the weak topology $\sigma\left(E, E^{\prime}\right)$, then $\left\{x^{(n)}\right\}_{n=1}^{\infty}$ converges in the topology $\lambda\left(E, E^{\prime}\right)$ of uniform convergence on $\sigma\left(E^{\prime}, E\right)$-compact sets.

Proof. Direct application of the general Orlicz-Pettis theorem (see [6], [15] or [21]).

Lemma 5. Let $E$ be a Fréchet space and suppose that $x^{(n)} \rightarrow x$ in $E$. Then there is a subsequence $\left\{z^{(n)}\right\}_{n=1}^{\infty}$ of $\left\{x^{(n)}\right\}_{n=1}^{\infty}$ that superconverges to $x$.

Proof. Suppose that $\left\{p_{k}\right\}_{k=1}^{\infty}$ is an increasing sequence of seminorms defining the topology on $E$. Choose an increasing sequence $\left\{n_{k}\right\}_{k=1}^{\infty}$ of positive integers so that

$$
p_{k}\left(x-x^{(n)}\right) \leq 1 / 2^{k}
$$

whenever $n \geq n_{k}$. Putting $z^{(k)}=x^{\left(n_{k}\right)}, k=1,2, \ldots$, it is easy to see that

$$
\sum_{k=1}^{\infty}\left(z^{(k)}-z^{(k-1)}\right)
$$


converges absolutely, so that $\left\{z^{(k)}\right\}_{k=1}^{\infty}$ superconverges to $x$ in $E$.

Lemma 6. $x^{(n)} \rightarrow x \sigma(m, l)$ if and only if $x^{(n)} \rightarrow x \sigma(m, \varphi)$ and $\sup _{n}\left\|x^{(n)}\right\|_{\infty}<\infty$.

Proof. A simple compactness argument (cf. [6, Lemma 3]). Alternatively, a neat proof may be given by using Lebesgue's dominated convergence theorem.

Lemma 7. If $x^{(n)} \rightarrow 0 \sigma\left(c_{0}, l\right)$, then there exists a subsequence $\left\{z^{(n)}\right\}_{n=1}^{\infty}$ of $\left\{x^{(n)}\right\}_{n=1}^{\infty}$ such that

$$
\left\|\frac{1}{n}\left(z^{(1)}+z^{(2)}+\cdots+z^{(n)}\right)\right\|_{\infty} \rightarrow 0 .
$$

Proof. In view of Lemma 6 the hypotheses are equivalent to

$$
\begin{array}{ll}
\lim _{j \rightarrow \infty} x_{j}^{(n)}=0 & (n=1,2, \ldots), \\
\lim _{n \rightarrow \infty} x_{j}^{(n)}=0 & (j=1,2, \ldots),
\end{array}
$$

and

$$
\sup _{n, j}\left|x_{j}^{(n)}\right|=M<\infty .
$$

We choose increasing sequences $\left\{s_{m}\right\}_{m=1}^{\infty}$ and $\left\{t_{m}\right\}_{m=1}^{\infty}$ of positive integers as follows. Let $s_{1}=1, t_{0}=0$, and suppose that $s_{1}, \ldots, s_{m}$ and $t_{1}, \ldots, t_{m-1}$ have been chosen. Using (7), choose $t_{m}>t_{m-1}$ so that

$$
\max _{1 \leq n \leq S_{m}}\left|x_{j}^{(n)}\right| \leq 2^{-m}
$$

whenever $j>t_{m}$. Next, using (8), choose $s_{m+1}>s_{m}$ so that

$$
\left|x_{j}^{\left(s_{m+1}\right)}\right| \leq 2^{-m}
$$

whenever $1 \leq j \leq t_{n}$.

If $t_{m}<j \leq t_{m+1}$ and $m \geq 1$, then

$$
\begin{aligned}
\mid x_{j}^{\left(s_{1}\right)}+x_{j}^{\left(s_{2}\right)}+\cdots+x_{j}^{\left(s_{n}\right)}, & \leq n \cdot 2^{-m} \text { if } n \leq m \text { by }(10), \\
& \leq m \cdot 2^{-m}+\left|x_{j}^{\left(s_{m+1}\right)}\right|+\sum_{k=m+1}^{n-1} 2^{-k} \\
& \quad \text { if } n>m \text { by }(10) \text { and }(11), \\
& \leq M+1 \text { by (9). }
\end{aligned}
$$

Consequently, putting $z^{(n)}=x^{\left(s_{n}\right)}, n=1,2, \ldots$, we have

$$
\begin{aligned}
\left\|\frac{z^{(1)}+z^{(2)}+\cdots+z^{(n)}}{n}\right\|_{\infty} & =\sup _{m} \sup _{\substack{i_{m}<j \leq i_{m+1}\\
}}\left|\frac{x^{\left(s_{1}\right)}+x_{j}^{\left(s_{2}\right)}+\cdots+x_{j}^{\left(s_{n}\right)}}{n}\right| \\
& \leq(M+1) / n \rightarrow 0 \text { as } n \rightarrow \infty .
\end{aligned}
$$


Lemma 7 says that in the Banach space $c_{0}$ every weakly convergent sequence has a subsequence whose arithmetic means converge in norm. This property, the so-called Banach-Saks property, is also known to hold for the spaces $l^{p}$ and $L^{P}(0,1)$ (see [3]). We remark here that not every Banach space has this property.

Lemma 8. Let $x^{(n)} \in c_{0}, n=1,2, \ldots$, and suppose that $x^{(n)} \rightarrow x \sigma(m, l)$. Then there exists a subsequence $\left\{z^{(n)}\right\}_{n=1}^{\infty}$ of $\left\{x^{(n)}\right\}_{n=1}^{\infty}$ that is superconvergent to $x$ in $\sigma(m, l)$.

Proof. Without loss of generality we may assume that $x=0$. The hypotheses are then the same as in Lemma 7 and we may choose $\left\{s_{n}\right\}_{n=1}^{\infty}$ and $\left\{t_{n}\right\}_{n=1}^{\infty}$ as before so that (9) and (10) are satisfied. It is easily seen that

$$
\sup _{j} \sum_{n=1}^{\infty}\left|x_{j}^{\left(s_{n}\right)}-x_{j}^{\left(s_{n-1}\right)}\right|<\infty,
$$

and so, in view of Lemma 6, we may take $z^{(n)}=x^{\left(s_{n}\right)}, n=1,2, \ldots$

Lemma 9. If $x \in a c_{0}$ and $\left\{s_{n}\right\}_{n=1}^{\infty}$ is a strictly increasing sequence of positive integers then the sequence

$$
y^{(n)}=\frac{1}{n}\left(P_{s_{1}} x+P_{s_{2}} x+\cdots+P_{s_{n}} x\right)
$$

is superconvergent to $x$ in $\sigma\left(a c_{0}, l\right)$.

Proof. We define

$$
\begin{aligned}
& Q_{0}=0 ; \quad Q_{n}=\frac{1}{n}\left(P_{s_{1}}+P_{s_{2}}+\cdots+P_{s_{n}}\right), \quad n=1,2, \ldots ; \\
& R_{n}=Q_{n}-Q_{n-1} .
\end{aligned}
$$

For any finite subset $M$ of the positive integers $\mathbf{Z}$ we write

$$
S_{M}=\sum_{k \in M} R_{k}=\sum_{k=1}^{\infty} \delta_{M}(k) R_{k}
$$

where $\delta_{M}$ is the characteristic function of $M$. We show that the collection $\left\{S_{M}: M\right.$ is a finite subset of $\left.Z\right\}$ is $\tau\left(a c_{0}, l\right) \rightarrow \tau\left(a c_{0}, l\right)$-equicontinuous. Since

$$
\sum_{k=1}^{\infty}\left(S_{M} f\right)_{k} x_{k}=\sum_{k=1}^{\infty} f_{k}\left(S_{M} x\right)_{k},
$$

we must show that if $C$ is $\sigma\left(l, a c_{0}\right)$-compact then $S(C)=\left\{S_{M} f: f \in C, M \subset \mathbf{Z}\right\}$ is $\sigma\left(l, a c_{0}\right)$-relatively compact.

For $x \in l$, we have

$$
\begin{aligned}
\left(Q_{n} x\right)_{p} & =(1-k / n) x_{p} & & \text { if } s_{k}<p \leq s_{k+1}, 1 \leq k \leq n, \\
& =0 & & \text { if } p>s_{n},
\end{aligned}
$$

and 


$$
\begin{aligned}
\left(R_{n} x\right)_{p} & =\frac{k}{n(n-1)} x_{p} & & \text { if } s_{k}<p<s_{k+1}, 1 \leq k \leq n-1, \\
& =0 & & \text { if } p>s_{n},
\end{aligned}
$$

so that

$$
\left(S_{M} x\right)_{p}=\left\{\sum_{n=k+1}^{\infty} \frac{k}{n(n-1)} \delta_{M}(n)\right\} x_{p} \quad \text { if } s_{k}<p \leq s_{k+1} .
$$

Now if $C$ is $\sigma\left(l, a c_{0}\right)$-relatively compact, then by Corollary 2 to Theorem 2, we have

$$
\sup _{x \in C}\|x\|_{h}=K<\infty
$$

and

$$
\sup _{x \in C} \sum_{p=n}^{\infty}\left|x_{p}-x_{p+1}\right|=\varepsilon_{n} \rightarrow 0
$$

Now

$$
\left|\left(S_{M} x\right)_{p}\right| \leq\left|x_{p}\right| \sum_{n=k+1}^{\infty} \frac{k}{n(n-1)} \leq\left|x_{p}\right|
$$

so that $\left\|S_{M} x\right\|_{\mathrm{h}} \leq K$. If $s_{k}<p<s_{k+1}$,

$$
\left(S_{M} x\right)_{p}-\left(S_{M} x\right)_{p+1}=\left(\sum_{n=k+1}^{\infty} \frac{k}{n(n-1)} \delta_{M}(k)\right)\left(x_{p}-x_{p+1}\right)
$$

so that

$$
\left|\left(S_{M} x\right)_{p}-\left(S_{M} x\right)_{p+1}\right| \leq\left|x_{p}-x_{p+1}\right|
$$

while, if $p=s_{k}$,

$$
\begin{aligned}
&\left(S_{M} x\right)_{p}-\left(S_{M} x\right)_{p+1}=\left\{\sum_{n=k}^{\infty} \frac{k-1}{n(n-1)} \delta_{M}(n)\right\} x_{p}-\left\{\sum_{n=k+1}^{\infty} \frac{k}{n(n-1)} \delta_{M}(n)\right\} x_{p+1} \\
&=\left\{\sum_{n=k}^{\infty} \frac{k-1}{n(n-1)} \delta_{M}(n)\right\}\left(x_{p}-x_{p+1}\right)-\left\{\sum_{n=k+1}^{\infty} \frac{1}{n(n-1)} \delta_{M}(n)\right\} x_{p+1} \\
&+\frac{1}{k} \delta_{M}(k) x_{p+1},
\end{aligned}
$$

so that

$$
\left|\left(S_{M} x\right)_{p}-\left(S_{M} x\right)_{p+1}\right| \leq\left|x_{p}-x_{p+1}\right|+\frac{1}{k}\left|x_{p+1}\right| .
$$

Consequently, if $s_{k} \leq n<s_{k+1}$, 


$$
\begin{aligned}
\sum_{p=n+1}^{\infty}\left|\left(S_{M} x\right)_{p}-\left(S_{M} x\right)_{p+1}\right| & \leq \frac{K}{k}+\sum_{p=n+1}^{\infty}\left|x_{p}-x_{p+1}\right| \\
& \leq \frac{K}{k}+\varepsilon_{n} \rightarrow 0 \text { as } n \rightarrow \infty .
\end{aligned}
$$

It follows from Corollary 2 to Theorem 2 that $S(C)$ is indeed $\sigma\left(l, a c_{0}\right)$-compact and so the collection $\left\{S_{M}: M \subseteq Z\right\}$ is equicontinuous on $\left(a c_{0}, \tau\left(a c_{0}, l\right)\right)$. In particular, if $N$ is an infinite subset of $\mathbf{Z}$, the operators

$$
\sum_{k=1}^{n} \delta_{N}(k) R_{k} \quad(n=1,2, \ldots)
$$

are equicontinuous, and so, since $a c_{0}$ is $\tau\left(a c_{0}, l\right)$-complete (Theorem 4(i)) the set of $x \in a c_{0}$ for which $\sum_{k=1}^{\infty} \delta_{N}(k) R_{k} x$ converges is closed. However if $x \in \varphi$ this is clearly so, and so we conclude for all $x \in a c_{0}$ and all $N \subseteq \mathbf{Z}$ that $\sum_{k=1}^{\infty} \delta_{N}(k) R_{k} x$ converges. Hence the sequence $\left\{Q_{n} x\right\}_{n=1}^{\infty}$ superconverges in $\left(a c_{0}, \tau\left(a c_{0}, l\right)\right)$.

Lemma 10. Let $x^{(n)} \in c_{0}, n=1,2, \ldots$, and suppose that $x^{(n)} \rightarrow x \sigma\left(a c_{0}, l\right)$. Then there exists a subsequence $\left\{z^{(n)}\right\}_{n=1}^{\infty}$ of $\left\{x^{(n)}\right\}_{n=1}^{\infty}$ such that some subsequence $\left\{w^{(n)}\right\}_{n=1}^{\infty}$ of $\left\{\left(z^{(1)}+z^{(2)}+\cdots+z^{(n)}\right) / n\right\}_{n=1}^{\infty}$ superconverges to $x$ in $\sigma\left(a c_{0}, l\right)$.

Proof. Since $P_{n} x \rightarrow x \sigma\left(a c_{0}, l\right)$, we have

$$
x^{(n)}-P_{n} x \rightarrow 0 \sigma\left(c_{0}, l\right) .
$$

By Lemma 7 we may take a subsequence $\left\{z^{(n)}\right\}_{n=1}^{\infty}=\left\{x^{\left(s_{n}\right)}\right\}_{n=1}^{\infty}$ of $\left\{x^{(n)}\right\}_{n=1}^{\infty}$ such that

$$
\left\|\frac{1}{n}\left(z^{(1)}+\cdots+z^{(n)}\right)-\frac{1}{n}\left(P_{s_{1}} x+\cdots+P_{s_{n}} x\right)\right\|_{\infty} \rightarrow 0 .
$$

Taking a subsequence again, we may suppose that, for each integer $n$,

$$
\left\|\frac{1}{m_{n}}\left(z^{(1)}+\cdots+z^{\left(m_{n}\right)}\right)-\frac{1}{m_{n}}\left(P_{s_{1}} x+\cdots+P_{s_{n}} x\right)\right\|_{\infty} \leq \frac{1}{2^{n}},
$$

so that the sequence

$$
\left\{\frac{1}{m_{n}}\left(z^{(1)}+\cdots+z^{\left(m_{n}\right)}\right)-\frac{1}{m_{n}}\left(P_{s_{1}} x+\cdots+P_{s_{m_{n}}} x\right)\right\}_{n=1}^{\infty}
$$

is superconvergent to 0 in $c_{0}$. However, by Lemmas 3 and 9 , the sequence

$$
\left\{\frac{1}{m_{n}}\left(P_{s_{1}} x+\cdots+P_{s_{m_{n}}} x\right)\right\}_{n=1}^{\infty}
$$

is superconvergent to $x$ in $\sigma\left(a c_{0}, l\right)$. Hence, with 


$$
w^{(n)}=\frac{1}{m_{n}}\left(z^{(1)}+\cdots+z^{\left(m_{n}\right)}\right) \quad(n=1,2, \ldots),
$$

$\left\{w^{(n)}\right\}_{n=1}^{\infty}$ is superconvergent to $x$ in $\sigma\left(a c_{0}, l\right)$.

Before stating our next result we recall the following notation. For an infinite matrix $A, a c_{A}$ denotes the set

$$
a c_{A}=\{x \in \omega: A x \in a c\} .
$$

If $x \in a c_{A}$, we write $\operatorname{Lim}_{A} x$ in place of $\operatorname{Lim}(A x)$, and denote by $\left(a c_{0}\right)_{A}$ the subspace of $(a c)_{A}$ on which $\operatorname{Lim}_{A}$ vanishes.

Theorem 6. Let $A$ be a matrix such that

(i) $\sup _{i} \sum_{j=1}^{\infty}\left|a_{i j}\right|<\infty$, and

(ii) $\lim _{i \rightarrow \infty} a_{i j}=0$ for $j=1,2, \ldots$ Then $l$ is $\sigma\left(l,\left(a c_{0}\right)_{A} \cap m\right)$-sequentially complete.

Proof. Let $x \in\left(a c_{0}\right)_{A} \cap m$ be fixed. We construct a sequence $\left\{z^{(n)}\right\}_{n=1}^{\infty}$ of elements of $\varphi$ such that $\left\{z^{(n)}\right\}_{n=1}^{\infty}$ superconverges to $x$ in $\sigma\left(\left(a c_{0}\right)_{A} \cap m, l\right)$. To do this, we first observe that

$$
A P_{n} x \rightarrow A x \quad \sigma(\omega, \varphi) .
$$

Condition (i) ensures that $A: m \rightarrow m$ is continuous and hence

$$
\left\|A P_{n} x\right\|_{b_{0}} \leq\|A\|\|x\|_{\infty} \text {. }
$$

Lemma 6 gives

$$
A P_{n} x \rightarrow A x \quad \sigma(m, l) .
$$

Now condition (ii) implies that $A P_{n} x \in A(\varphi) \subset c_{0}$ so we may apply Lemma 10 to deduce the existence of a sequence $\left\{v^{(k)}\right\}_{k=1}^{\infty}$ such that $\left\{A v^{(k)}\right\}_{k=1}^{\infty}$ superconverges to $A x$ in $\sigma\left(a c_{0}, l\right)$ and $\left\{v^{(k)}\right\}_{k=1}^{\infty}$ takes the form

$$
v^{(k)}=\frac{1}{m_{k}}\left(u^{(1)}+\cdots+u^{\left(m_{k}\right)}\right)
$$

where $\left\{u^{(k)}\right\}_{k=1}^{\infty}$ is some subsequence of $\left\{P_{n} x\right\}_{n=1}^{\infty}$. Clearly we have

$$
\sup _{k}\left\|v^{(k)}\right\|_{\infty} \leq\|x\|_{\infty}
$$

and $v^{(k)} \rightarrow x \sigma(\omega, \varphi)$ so that $v^{(k)} \rightarrow x \sigma(m, l)$ by Lemma 6 .

Furthermore, since $A v^{(k)} \rightarrow A x$ in $\omega$, we have

$$
v^{(k)} \rightarrow x \text { in } \omega_{A} \text {. }
$$

We now apply Lemmas 3,5 and 8 to obtain a subsequence $\left\{z^{(n)}\right\}_{n=1}^{\infty}$ of $\left\{u^{(n)}\right\}_{n=1}^{\infty}$ such that $\left\{z^{(n)}\right\}_{n=1}^{\infty}$ superconverges to $x$ in both $\omega_{A}$ and $\sigma(m, l)$; it is also clear that 
$\left\{A z^{(n)}\right\}_{n=1}^{\infty}$ superconverges in $\left(a c_{0}, \sigma\left(a c_{0}, l\right)\right)$. Now suppose that $\left\{\varepsilon_{n}\right\}_{n=1}^{\infty}$ is a sequence taking only the values 1 and 0 ; for each $k$ let

$$
y_{k}=\sum_{n=1}^{\infty} \varepsilon_{n}\left(z_{k}^{(n)}-z_{k}^{(n-1)}\right) \quad\left(\text { where } z^{(0)}=0\right) .
$$

Since $\left\{z^{(n)}\right\}_{n=1}^{\infty}$ superconverges in both $\omega_{A}$ and $(m, \sigma(m, l))$, the series

$$
\sum_{n=1}^{\infty} \varepsilon_{n}\left(z^{(n)}-z^{(n-1)}\right)
$$

converges to $y$ in both $\omega_{A}$ and $(m, \sigma(m, \eta))$; therefore $y \in m \cap \omega_{A}$. Now $A: \omega_{A} \rightarrow \omega$ is continuous [24, Theorem $\left.4.4(\mathrm{c})\right]$ and so

$$
A y=\sum_{n=1}^{\infty} \varepsilon_{n}\left(A z^{(n)}-A z^{(n-1)}\right) \quad \sigma(\omega, \varphi)
$$

However, $\left\{A z^{(n)}\right\}_{n=1}^{\infty}$ superconverges in $\left(a c_{0}, \sigma\left(a c_{0}, l\right)\right)$ so that

$$
A y=\sum_{n=1}^{\infty} \varepsilon_{n}\left(A z^{(n)}-A z^{(n-1)}\right) \quad \sigma\left(a c_{0}, l\right),
$$

and $A y \in a c_{0}$, i.e., $y \in\left(a c_{0}\right)_{A}$. Thus $\left\{z^{(n)}\right\}_{n=1}^{\infty}$ superconverges to $x$ in $\left(\left(a c_{0}\right)_{A} \cap m\right.$, $\left.\sigma\left(\left(a c_{0}\right)_{A} \cap m, l\right)\right)$.

We now repeat the argument used in the proof of Theorem 3 of [6]. Consider the topology $\lambda\left(\left(a c_{0}\right)_{A} \cap m, l\right)$ on $\left(a c_{0}\right)_{A} \cap m$ of uniform convergence on the $\sigma\left(l,\left(a c_{0}\right)_{A} \cap m\right)$-compact subsets of $l$; by Lemma 4 we have

$$
z^{(n)} \rightarrow x \quad \lambda\left(\left(a c_{0}\right)_{A} \cap m, l\right) .
$$

Suppose now that $\psi$ is a linear functional on $\left(a c_{0}\right)_{A} \cap m$ whose restrictions to $\lambda\left(\left(a c_{0}\right)_{A} \cap m, l\right)$-precompact sets are $\lambda$-continuous. Then $\psi$ is $\lambda$-sequentially continuous and since $\lambda \leq \beta\left(\left(a c_{0}\right)_{A} \cap m, l\right) \leq \beta\left(c_{0}, l\right), \psi$ is $\|\cdot\|_{b_{0}}$-continuous on $c_{0}$ so that

$$
\sum_{j=1}^{\infty}\left|f_{j}\right|<\infty
$$

where $\psi\left(e^{(j)}\right)=f_{j}(j=1,2, \ldots)$. Now

$$
\psi\left(z^{(n)}\right)=\sum_{j=1}^{\infty} z_{j}^{(n)} f_{j}
$$

since $z^{(n)} \in \varphi$, and

$$
\lim _{n \rightarrow \infty} \sum_{j=1}^{\infty} z_{j}^{(n)} f_{j}=\sum_{j=1}^{\infty} x_{j} f_{j}
$$

since $z^{(n)} \rightarrow x \sigma(m, l)$. Consequently, for each $x \in\left(a c_{0}\right)_{A} \cap m$, we have

$$
\psi(x)=\sum_{j=1}^{\infty} x_{j} f_{j}
$$


It follows (as in the proof of Theorem 3 of [6]) by Grothendieck's completeness theorem that the topology $\rho$ on $l$, of uniform convergence on $\lambda$-precompact subsets of $\left(a c_{0}\right)_{A} \cap m$, must be complete. Furthermore, $\rho$ defines the same convergent and Cauchy sequences as $\sigma\left(l,\left(a c_{0}\right)_{A} \cap m\right)$ so that $l$ is $\sigma\left(l,\left(a c_{0}\right)_{A} \cap m\right)$ sequentially complete.

We now come to our first consistency theorem.

Theorem 7. Let $A$ and $B$ be regular matrices and suppose that $a c_{A} \cap m \subset c_{B}$. Then $\lim _{B} x=\operatorname{Lim}_{A} x$ whenever $x \in a c_{A} \cap m$.

Proof. Since $A$ is regular, the conditions of Theorem 6 are satisfied. Let $b^{(n)} \in l, n=1,2, \ldots$, be defined by

$$
b_{k}^{(n)}=b_{n k} \quad(k=1,2, \ldots),
$$

(so that $b^{(n)}$ is the $n$th row of $B$ ). Since $\left(a c_{0}\right)_{A} \cap m \subseteq c_{B}$, we have

$$
\lim _{n \rightarrow \infty} \sum_{k=1}^{\infty} b_{k}^{(n)} x_{k} \text { exists }
$$

whenever $x \in\left(a c_{0}\right)_{A} \cap m$. Hence $\left\{b^{(n)}\right\}_{n=1}^{\infty}$ is $\sigma\left(l,\left(a c_{0}\right)_{A} \cap m\right)$-Cauchy and so converges, say $b^{(n)} \rightarrow b$, by Theorem 6 . Clearly

$$
b_{k}=\lim _{k \rightarrow \infty} b_{n k}=0,
$$

so that $b^{(n)} \rightarrow 0 \sigma\left(l,\left(a c_{0}\right)_{A} \cap m\right)$.

Now, if $x \in(a c)_{A} \cap m$, then $x-\left(\operatorname{Lim}_{A} x\right) e \in\left(a c_{0}\right)_{A} \cap m$, and so

$$
\lim _{B}(x-(\operatorname{Lim} x) e)=\lim _{n \rightarrow \infty} \sum_{k=1}^{\infty} b_{k}^{(n)}\left(x_{k}-\operatorname{Lim}_{A} x\right)=0,
$$

i.e. $\lim _{B} x=\operatorname{Lim}_{A} x$.

When $A$ is the identity matrix, Theorem 7 reduces to the following.

Corollary. Let $B$ be a regular matrix with ac $\subset c_{B}$. Then $\lim _{B} x=\operatorname{Lim} x$ whenever $x \in a c$.

This special result may also be derived from Corollary 2 to Theorem 5 and was first obtained by Lorentz [13].

Before stating our next result let us recall the following notation. If $E$ is an $F K$ space containing $\varphi$, then we write

$$
W_{E}=\left\{x \in E: P_{n} x \rightarrow x \text { weakly in } E\right\}
$$

and

$$
S_{E}=\left\{x \in E: P_{n} x \rightarrow x \text { in } E\right\}
$$

Theorem 8. Let $E$ be an $F K$-space containing $c_{0}$. Then $l$ is sequentially complete 
under both the topologies $\sigma\left(l, W_{E} \cap a c_{0}\right)$ and $\sigma\left(l, S_{E} \cap a c_{0}\right)$.

Proof. As with Theorem 6, the proof hinges on ideas developed in Theorem 3 of [6].

Let $x \in W_{E} \cap a c_{0}$ be fixed: by Theorem 2 of [6] (see also [20]) there is a sequence $\left\{u^{(n)}\right\}_{n=1}^{\infty}$ of elements of $\varphi$ with

$$
\tau-\lim _{n \rightarrow \infty} u^{(n)}=x
$$

and

$$
\sup _{n}\left\|u^{(n)}\right\|_{b_{0}} \leq\|x\|_{b_{0}},
$$

where $\tau$ denotes the $F K$-topology on $E$. By Lemma 6,

$$
\lim _{n \rightarrow \infty} u^{(n)}=x \quad \sigma\left(a c_{0}, l\right)
$$

and so, by Lemma 10 , there exists a sequence $\left\{v^{(n)}\right\}_{n=1}^{\infty}$, of arithmetric means of a subsequence of $\left\{u^{(n)}\right\}_{n=1}^{\infty}$, such that $\left\{v^{(n)}\right\}_{n=1}^{\infty}$ superconverges to $x$ in $\sigma\left(a c_{0}, l\right)$; clearly

$$
\tau-\lim _{n \rightarrow \infty} v^{(n)}=x .
$$

By using Lemmas 3 and 5 we may select a subsequence $\left\{z^{(n)}\right\}_{n-1}^{\infty}$ which superconverges to $x$ in both $\tau$ and $\sigma\left(a c_{0}, l\right)$. Thus every subseries of $\sum_{n=1}^{\infty}\left(z^{(n)}-z^{(n-1)}\right)$ converges in $E \cap a c_{0}$; i.e., if $\epsilon_{n}=0$ or 1 for all $n$ and

$$
y=\sum_{n=1}^{\infty} \varepsilon_{n}\left(z^{(n)}-z^{(n-1)}\right) \quad\left(\text { where } z^{(0)}=0\right),
$$

then $y \in E \cap a c_{0}$. Since this series converges in $\sigma(m, l)$, we have

$$
\sup _{k}\left\|\sum_{n=1}^{k} \varepsilon_{n}\left(z^{(n)}-z^{(n-1)}\right)\right\|_{\infty}<\infty,
$$

and since the series converges in $\tau$ we have $y \in W_{E}$ by Theorem 2 of [6]. Thus $\left\{z^{(n)}\right\}_{n=1}^{\infty}$ superconverges to $x$ in $\sigma\left(W_{E} \cap a c_{0}, l\right)$, and the remaining details follow those of Theorem 6 (or Theorem 3 of [6]).

For the second half of the theorem we observe that [10, pp. 1015-1016] $S_{E}=W_{F}$, where $F$ is the $F K$-space defined as follows.

$$
F=\left\{x \in E:\left\{P_{n} x\right\}_{n=1}^{\infty} \text { is } \tau \text {-bounded }\right\}
$$

with the topology given by the seminorms

$$
\nu(x)=\sup _{n} \nu\left(P_{n} x\right) \quad(x \in F)
$$

for each $\tau$-continuous seminorm $\nu$.

Our next result may be thought of as a generalized consistency theorem. 
Theorem 9. Let $E$ be an $F K$-space containing $c_{0}$ and let $F$ be an $F K$-space containing no (closed) subspace isomorphic to $m$. If $W_{E} \cap a c_{0} \subset F$, then $W_{E} \cap a c_{0}$ $\subset W_{F}$.

Proof. As in the proof of Theorem 8 we can show that each $x \in W_{E} \cap a c_{0}$ can be written in the form $x=\sum_{n=1}^{\infty} x^{(n)}$ where $x^{(n)} \in \varphi, n=1,2, \ldots$, and the convergence is $\sigma\left(W_{E} \cap a c_{0}, l\right)$-subseries. This observation enables us to replace $W_{E} \cap m$ in the statement of Proposition 1 of [8] by $W_{E} \cap a c_{0}$; but then the present result follows just as in the proof of Theorem 2 of [8].

In particular, it should be noted that Theorem 9 remains valid when $F$ is a separable $F K$-space.

Theorem 10. Let $A$ and $B$ be regular matrices and suppose that $a c \cap c_{A} \subseteq c_{B}$. Then there exists a constant $\alpha$ such that

$$
\lim _{B} x=\alpha \lim _{A} x+(1-\alpha) \operatorname{Lim} x
$$

whenever $x \in a c \cap c_{A}$.

Proof. Since $A$ is regular we have, by Theorem 3.6 of [25], $\left(c_{0}\right)_{A} \cap a c_{0}$ $=W_{c_{A}} \cap a c_{0}$. Now $c_{B}$ is separable ([14], [4]) so that

$$
\left(c_{0}\right)_{A} \cap a c_{0} \subset W_{c_{B}} \cap a c_{0}=\left(c_{0}\right)_{B} \cap a c_{0}
$$

by Theorem 9. Hence $\lim _{A} x=\operatorname{Lim} x=0$ implies that $\lim _{B} x=0$, and so

$$
\lim _{B} x=\alpha \lim _{A} x+\beta \operatorname{Lim} x
$$

whenever $x \in a c \cap c_{A}$. However $1=\lim _{B} e=\alpha+\beta$ and the desired conclusion follows.

Theorem 11. Let $A$ and $B$ be conservative matrices and suppose that ac $\cap c_{A}$ $\subseteq c_{B}$. Then there exist constants $\alpha, \beta$ such that

(i) $\lim _{B} x-\sum_{j=1}^{\infty} b_{j} x_{j}=\alpha\left(\lim _{A} x-\sum_{j=1}^{\infty} a_{j} x_{j}\right)+\beta \operatorname{Lim} x$ whenever $x \in a c$ $\cap c_{A}$, and

(ii) $\chi(B)=\alpha \chi(A)+\beta$.

Proof. This is a simple extension of Theorem 10; we observe that

$$
W_{c_{A}} \cap a c_{0}=\left\{x: \lim _{\boldsymbol{A}} x=\sum_{j=1}^{\infty} a_{j} x_{j}\right\} \cap a c_{0}
$$

and apply the same method.

Corollary 1. Let $A$ be conull and $B$ be regular and suppose that ac $\cap c_{A} \subseteq c_{B}$. Then there exists a constant $\alpha$ such that

$$
\lim _{B} x=\operatorname{Lim} x+\alpha\left(\lim _{A} x-\sum_{j=1}^{\infty} a_{j} x_{j}\right)
$$


whenever $x \in$ ac $\cap c_{A}$.

Corollary 2. Let $A$ be regular and $B$ be conull and suppose that ac $\cap c_{A} \subseteq c_{B}$. Then there exists a constant $\alpha$ such that

$$
\lim _{B} x=\alpha\left(\lim _{A} x-\operatorname{Lim} x\right)+\sum_{j=1}^{\infty} b_{j} x_{j}
$$

whenever $x \in$ ac $\cap c_{A}$.

\section{REFERENCES}

1. A. Alexiewicz and Z. Semadeni, Linear functionals on two-norm spaces, Studia Math. 17 (1958), 121-140. MR 20 \#6644.

2. S. Banach, Théorie des operations linéaires, Monografie Mat., PWN, Warsaw, 1932.

3.S. Banach and S. Saks, Sur la convergence forte dans les champs $L^{P}$, Studia Math. 2 (1930), 51-57.

4. G. Bennett, $A$ representation theorem for summability domains, Proc. Lond. Math. Soc. (3) 24 (1972), 193-203. MR 45 \# 776.

5. - A new class of sequence spaces with applications in summability theory, J. Reine Angew. Math. 266 (1974), 49-75.

6. G. Bennett and N.J. Kalton, FK-spaces containing $c_{0}$, Duke Math. J. 39 (1972), 561-582.

7.—, Inclusion theorems for $K$-spaces, Canad. J. Math. (to appear).

8. - Addendum to FK-spaces containing $c_{0}$, Duke Math. J. 39 (1972), 819-821.

9. M.M. Day, Normed linear spaces, 2nd rev. ed., Academic Press, New York; Springer-Verlag, Berlin, 1962. MR 26 \#2847.

10. D.J.H. Garling, On topological sequence spaces, Proc. Cambridge Philos. Soc. 63 (1967), 997-1019. MR 36 \# 1964.

11. N.J. Kalton, Some forms of the closed graph theorem, Proc. Cambridge Philos. Soc. 70 (1971), 401-408.

12. J.P. King, Almost summable sequences, Proc. Amer. Math. Soc. 17 (1966), 1219-1225. MR 34 \#1752.

13. G.G. Lorentz, A contribution to the theory of divergent sequences, Acta Math. 80 (1948), 167-190. MR 10, 367.

14. S. Mazur and W. Orlicz, On linear methods of summability, Studia Math. 14 (1954), 129-160. MR 16, 814.

15. C.W. McArthur, On a theorem of Orlicz and Pettis, Pacific J. Math. 22 (1967), 297-302. MR 35 \#4702.

16. C.W. McArthur and J.R. Retherford, Uniform and equicontinuous Schauder bases of subspaces, Canad. J. Math. 17 (1965), 207-212. MR 30 \#4141.

17. G.M. Petersen, Almost convergence and the Buck-Pollard property, Proc. Amer. Math. Soc. 11 (1960), 469-477. MR 22 \#2819.

18. H.H. Schaefer, Topological vector spaces, Macmillan, New York, 1966. MR 33 \# 1689.

19. P. Schaefer, Almost convergent and almost summable sequences, Proc. Amer. Math. Soc. 20 (1969), 51-54. MR 38 \#3649.

20. A.K. Snyder, Conull and coregular FK-spaces, Math. Z. 90 (1965), 376-381. MR 32 \#2783.

21. I. Tweddle, Vector-valued measures, Proc. London Math. Soc. (3) 20 (1970), 469-489. MR 41 \#3707.

22. A. Wilansky, Topics in functional analysis, Lecture Notes in Math., no. 45, Springer-Verlag, Berlin and New York, 1967. MR 36 \#6901.

23. A. Wiweger, Linear spaces with mixed topology, Studia Math. 20 (1961), 47-68. MR 24 \#A3490.

24. K. Zeller, Allgemeine Eigenschaften von Limitierungsverfahren, Math. Z. 53 (1951), 463-487. MR 12, 604 . 
25.—, Abschnittskonvergenz in FK-Rüumen, Math. Z. 55 (1951), 55-70. MR 13, 934.

26. K. Zeller and W. Beekman, Theorie der Limitierungsverfahren. Zweite, erweiterte und verbesserte Auflage, Ergebnisse der Math. und ihrer Grenzgebiete, Band 15, Springer-Verlag, Berlin and New York, 1970. MR 41 \#8863.

DEPARTMENT OF MATHEMATICS, INDIANA UNIVERSITY, BLOOMINGTON, INDIANA 47401

DEPARTMENT OF PURE MATHEMATICS, UNIVERSITY COLLEGE OF SWANSEA, SWANSEA SA2 8PP, WALES 